\title{
Detection of nonthermal emission from the bow shock of a massive runaway star
}

\author{
P. Benaglia ${ }^{1,2, \star}$, G. E. Romero ${ }^{1,2, \star}$, J. Marti ${ }^{3}$, C. S. Peri ${ }^{1,2, \star \star}$, and A. T. Araudo ${ }^{1,2, \star \star}$ \\ 1 Instituto Argentino de Radioastronomía, CCT-La Plata (CONICET), C.C.5, 1894 Villa Elisa, Argentina \\ e-mail: [pbenaglia;cperi]@fcaglp.unlp.edu.ar, [romero; anabella]@iar-conicet.gov.ar \\ 2 Facultad de Cs. Astronómicas y Geofísicas, UNLP, Paseo del Bosque s/n, 1900 La Plata, Argentina \\ 3 Departamento de Física (EPS), Universidad de Jaén, Campus Las Lagunillas s/n, Edif. A3, 23071 Jaén, Spain \\ e-mail: jmarti@ujaen.es
}

Received 18 June 2010 / Accepted 17 July 2010

\section{ABSTRACT}

\begin{abstract}
Context. The environs of massive, early-type stars have been inspected in recent years in the search for sites where particles can be accelerated up to relativistic energies. Wind regions of massive binaries that collide have already been established as sources of highenergy emission; however, there is a different scenario for massive stars where strong shocks can also be produced: the bow-shaped region of matter piled up by the action of the stellar strong wind of a runaway star interacting with the interstellar medium.

Aims. We study the bow-shock region produced by a very massive runaway star, BD $+43^{\circ} 3654$, to look for nonthermal radio emission as evidence of a relativistic particle population.

Methods. We observed the field of $\mathrm{BD}+43^{\circ} 3654$ at two frequencies, 1.42 and $4.86 \mathrm{GHz}$, with the Very Large Array (VLA), and obtained a spectral index map of the radio emission.

Results. We have detected, for the first time, nonthermal radio emission from the bow shock of a massive runaway star. Conclusions. After analyzing the radiative mechanisms that can be at work, we conclude that the region under study could produce enough relativistic particles whose radiation might be detectable by forthcoming gamma-ray instruments, like CTA North.
\end{abstract}

Key words. stars: early-type - stars: individual: $\mathrm{BD}+43^{\circ} 3654$ - radio continuum: general - infrared: stars

\section{Introduction}

Early-type stars with high peculiar velocities (i.e. runaway stars, with velocities $v_{*}>30 \mathrm{~km} \mathrm{~s}^{-1}$, e.g. Gies \& Bolton 1986) are uncommon. For instance, Maíz-Apellániz et al. (2004, Galactic O star catalog for $V<8$ stars) list $\sim 8 \%$ runaway stars out of 370 . These particular stars can be identified by the perturbation that they produce in the ambient medium (e.g. Kobulnicky et al. 2010, and references therein). When the strong winds of runaway OB stars sweep relatively large amounts of gas and dust, the material piles up in the so-called stellar bow shock. Bow shocks develop as arc-shaped structures, with bows pointing in the same direction as the stellar velocity, while the star moves supersonically in the surrounding interstellar medium (ISM). The winds are confined by the ram pressure of the ISM, at distances from the star determined by momentum balance. The stellar and shock-excited radiation heats the dust and gas swept by the bow shock. The dust, in turn, re-radiates the energy as mid-to-far IR excess flux.

As soon as IRAS images became available, Van Buren \& McCray (1988) looked for bow-shaped features near high-velocity $\mathrm{O}$ stars (see also Van Buren et al. 1995; Noriega-Crespo et al. 1997). The authors detected an IR candidate close to the $\mathrm{O}$ supergiant $\mathrm{BD}+43^{\circ} 3654\left(\alpha, \delta[\mathrm{J} 2000]=20^{\mathrm{h}} 33^{\mathrm{m}} 36.077^{\mathrm{s}},+43^{\circ} 59^{\prime} 07.40^{\prime \prime}\right.$; $\left.l, b=82.41^{\circ},+2.33^{\circ}\right)$.

\footnotetext{
$\star$ Member of CONICET.

$\star \star$ Fellow of CONICET.
}

Recently, Comerón \& Pasquali (2007) related the star $\mathrm{BD}+43^{\circ} 3654$ to a bow shock detected with the Midcourse Space eXperiment (MSX) at $D$ and $E$ bands. They studied the stellar motion relative to the surrounding material and proposed the star is a runaway member from Cyg OB2 association. Comerón and Pasquali determined a spectral type O4 If, and derived an age of about 1.6 Myr and a stellar mass of $\sim 70 M_{\odot}$, which makes the star one of the three more massive runaway stars known so far. On the basis of these estimates, the authors favor a dynamical ejection scenario (see Hoogerwerf et al. 2000, 2001, for reviews about the origin of runaway stars).

Gvaramadze \& Bomans (2008) suggest instead that $\mathrm{BD}+43^{\circ} 3654$ is part of a stellar system, formed by a close encounter between two tight massive binaries in the core of Cyg OB2. The star should be a blue straggler to match the timescales involved in their hypothesis. Kobulnicky et al. (2010) measured a heliocentric radial velocity of $-66.2 \pm 9.4 \mathrm{~km} \mathrm{~s}^{-1}$, and derived a stellar mass-loss rate of $1.6 \times 10^{-4} M_{\odot} \mathrm{yr}^{-1}$.

We analyzed data from the NRAO-VLA Sky Survey (NVSS, Condon et al. 1998). The images revealed a coma-shaped source of $\sim 7$ arcmin, spatially coincident with the MSX structure (see Fig. 1; NVSS angular resolution: $45^{\prime \prime}$; rms noise: $1 \mathrm{mJy} \mathrm{beam}^{-1}$ ). No point sources above $5 \sigma(40 \mathrm{mJy})$ that are positionally coincident with the MSX source are detected in the MIT-Green Bank Survey (GB6, Griffith et al. 1991). Inspection of the continuum emission at 408 and $1420 \mathrm{MHz}$ with the CGPS Survey (Taylor et al. 2003, angular resolutions of 3.4 and 1') confirms that the region centered on Cyg OB2 is complex and has strong emission on various angular scales (Peri et al. 2010). 


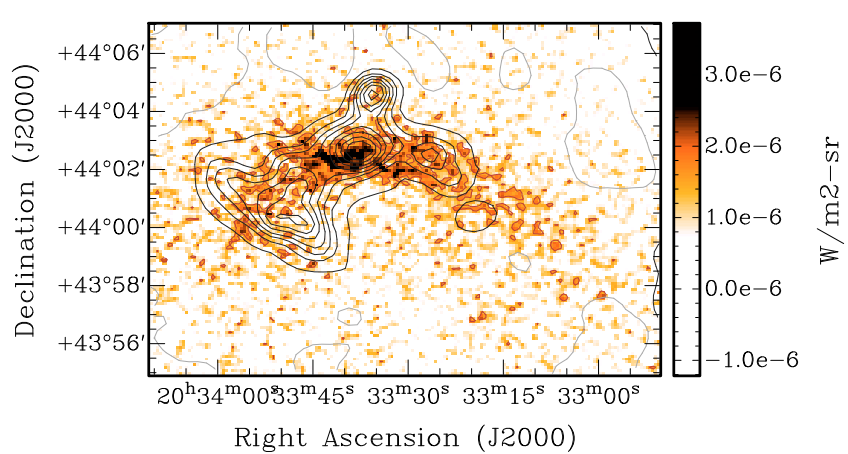

Fig. 1. MSX-D band image (color scale) superposed to $1.4 \mathrm{GHz}-\mathrm{NVSS}$ contours. Levels are: $-2,2(2 \sigma), 5,8,11,15,19,24,29,50,70$, and $90 \mathrm{mJy}^{\text {beam }}{ }^{-1}$.

A radio study of the bow shock can shed light on the physical processes that give rise to high-energy emission from a stellar source, regardless of the history of the runaway star. The shock can accelerate particles up to relativistic energies by Fermi mechanism. Energetic electrons will cool through synchrotron radiation, producing a nonthermal radio source. We carried out radio observations at two frequencies to study the nature of the emission from the bow shock of $\mathrm{BD}+43^{\circ} 3654$.

In this Letter we present the results of the radio observations in the form of a spectral index map of the bow-shock region of the runaway star and distinguish between thermal and nonthermal emission regions. We briefly discuss the issue of whether a bow shock could produce high-energy emission enough to be detected with instruments like Fermi or the future Cherenkov Telescope Array (CTA) and the conditions that must be fulfilled to achieve detection.

\section{Observations and results}

We carried out continuum observations with the Very Large Array $\left(\mathrm{NRAO}^{1}\right)$ in two array configurations: $\mathrm{C}$ at $1.42 \mathrm{GHz}$ in April 2008 and D at $4.86 \mathrm{GHz}$ in August 2008. The set up allowed mapping largest structures of the size of the MSX source and also guaranteed good matching beams of $12^{\prime \prime}$. The flux calibrator used was $0137+331^{2}$. Phase calibrator scans $-2052+365$ at $L$ band and 2007+404 at $C$ band-were interleaved with target scans. The total time on source was $3 \mathrm{~h}$ at each band and $50 \mathrm{MHz}$ for the total bandwidth.

The data was calibrated with the AIPS package in the standard way and analyzed with the Miriad routines. We used imagr to produce robust-weighted images. Figure 2 presents the resulting images after primary beam correction, re-gridded with the same synthesized beam. At both frequencies there is emission along the extension of the MSX source. The radio source is larger at $1.42 \mathrm{GHz}$, toward the eastern half of IR contours of the bow shock (increasing right ascensions). Clearly, the spectral index $\alpha\left(S_{v} \propto v^{\alpha}\right)$ is negative in there. In both images, the rms attained is similar $\left(0.3 \mathrm{mJy}^{-1}\right.$ beam ${ }^{-1}$ at $1.42 \mathrm{GHz}$ and 0.2 at 4.8 , $12^{\prime \prime}$ synthesized beam).

A detached ellipsoidal source at $\alpha, \delta[\mathrm{J} 2000]=$ $20^{\mathrm{h}} 33^{\mathrm{m}} 35^{\mathrm{s}}, 44^{\circ} 04^{\prime} 30^{\prime \prime}$, called ES here, is the strongest one in the field. The fluxes are $S_{\mathrm{ES}}(1.4 \mathrm{GHz})=105 \pm 10 \mathrm{mJy}$ and

\footnotetext{
1 The National Radio Astronomy Observatory is a facility of the National Science Foundation operated under cooperative agreement by Associated Universities, Inc.

${ }^{2}$ Calibrator details can be found at www.vla.nrao.edu/
}
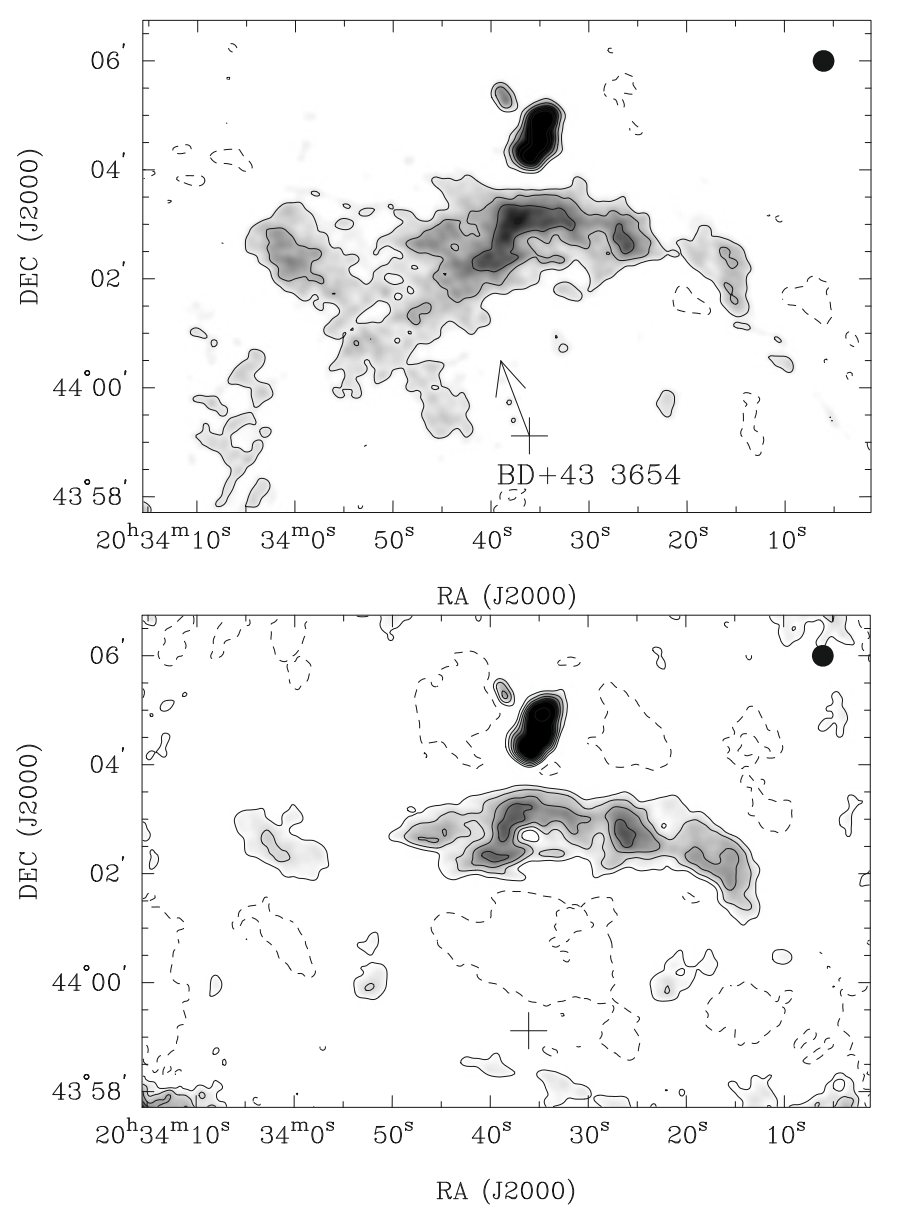

Fig. 2. Continuum emission at $1.42 \mathrm{GHz}$ (upper panel), and at $4.86 \mathrm{GHz}$ (lower panel). Contour levels are $-3,3,6,10,15,20,25$, and 60 times the rms of 0.3 and 0.2 mJy beam ${ }^{-1}$. BD $+43^{\circ} 3654$ is marked with a cross. The arrow represents the velocity of the star, derived from proper motions corrected for local motion of the surrounding ISM (see text). Synthesized beams of $12^{\prime \prime} \times 12^{\prime \prime}$ are shown in the top right corners.

$S_{\mathrm{ES}}(4.8 \mathrm{GHz})=95 \pm 5 \mathrm{mJy} . \mathrm{ES}$ spectral index $\langle\alpha\rangle=-0.1 \pm 0.1$ is characteristic of an optically thin HII region.

The hypothesis of a physical association between the star and the radio/IR features is supported by the very good agreement of the residual proper motion of the star $\left(15.7^{\circ} \pm 9.4^{\circ}\right.$ east of north, see Comerón \& Pasquali 2007) and the direction from the star to the apsis of the bow shock $\left(8.8^{\circ} \pm 10^{\circ}\right.$ east of north). The star velocity vector on the plane of the sky is represented in the upper panel of Fig. 2.

We used the continuum images at 1.42 and $4.86 \mathrm{GHz}$ to build a spectral index distribution map. We only considered input pixels with a signal-to-noise ratio $\geq 4$. Besides this, the spectral index map was masked for a signal-to-noise ratio $\geq 10$. Figure 3 shows the spectral index distribution and corresponding noise maps.

\section{Nonthermal radio emission from the bow shock}

In Fig. 3 we have represented the spectral index values derived from 1.42 and $4.86 \mathrm{GHz}$ data at positions where (i) the radio continuum signals are well above the noise (4 times or more); and (ii) the values of the spectral index errors are low $(<10 \%)$. It can be appreciated from the figure that there is a significant fraction of the portrayed area that shows negative spectral indices. 

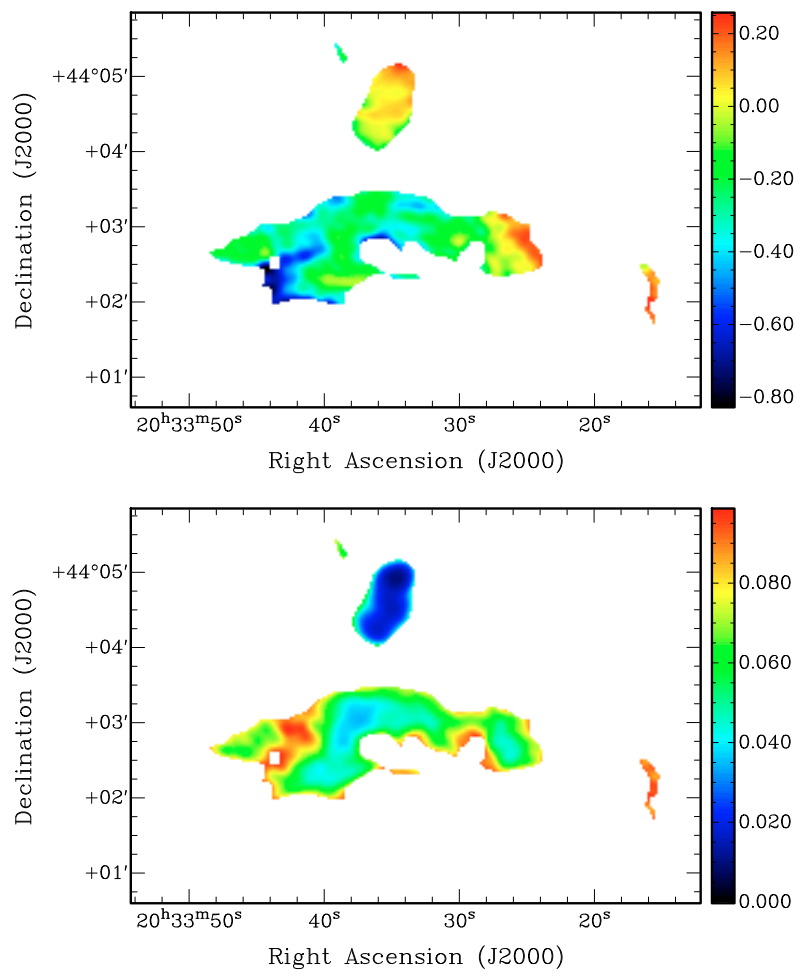

Fig. 3. Top: spectral index distribution. Bottom: spectral index error distribution.

The $\alpha$-values range from -0.8 to +0.3 , approximately from $\mathrm{W}$ to E. The source ES, in contrast, is characterized by $\alpha \rightarrow 0$, and it looks unrelated to the bow-shock feature.

In what follows, we assume that the MSX-D\&E bands excess emission represents the projection of the bow shock in the plane of the sky. We adopt a distance to the bow shock of $1.4 \mathrm{kpc}$ (Hanson 2003; Comerón \& Pasquali 2007). We measured a distance from the star $\mathrm{BD}+43^{\circ} 3654$ to the bow shock $R=5^{\prime}$, or $2 \mathrm{pc}$ (at $1.4 \mathrm{kpc}$ ). The shape of the bow shock can be fitted to a spherical cap of height $2^{\prime}=0.8 \mathrm{pc}$. We approximated the area of the MSX structure on the plane of the sky by $6^{\prime}$ in length times $2^{\prime}$ in height. If the depth is equal to the length, the volume occupied by the bow shock is $\sim 4.6 \mathrm{pc}^{3}$. Kobulnicky et al. (2010) suggest a conservative lower limit for the particle density in the bow-shock region of $100 \mathrm{~cm}^{-3}$. The area circumscribed by the projection of the cap is $\sim 1.8 \mathrm{pc}^{2}$. Most of this area shows a source of nonthermal radiation with averaged index $\alpha=-0.5$. Such a radiation is expected from synchrotron emission generated by relativistic electrons accelerated either at the forward shock in the ISM or in the reverse shock in the stellar wind. Since the latter is expected to have velocities $v_{\mathrm{rs}} \sim v_{\mathrm{w}} \approx 2300 \mathrm{~km} \mathrm{~s}^{-1}$, it should be more efficient for electron acceleration (as the efficiency $\eta \propto\left(v_{\mathrm{rs}} / c\right)^{2}$ for first-order Fermi-like diffusive acceleration, e.g. Drury 1983). The forward shock moves in a denser medium with lower velocities, $\sim 400 \mathrm{~km} \mathrm{~s}^{-1}$, but it might be effective to accelerate electrons as well although only up to lower energies.

To estimate the number density of relativistic particles, we followed the standard method by using the observed flux density and spectral slope, along with the hypothesis of equipartition between magnetic field and relativistic particles energy density (e.g. Ginzburg \& Syrovatskii 1964; Araudo et al. 2007). We considered that the energy density of relativistic particles makes

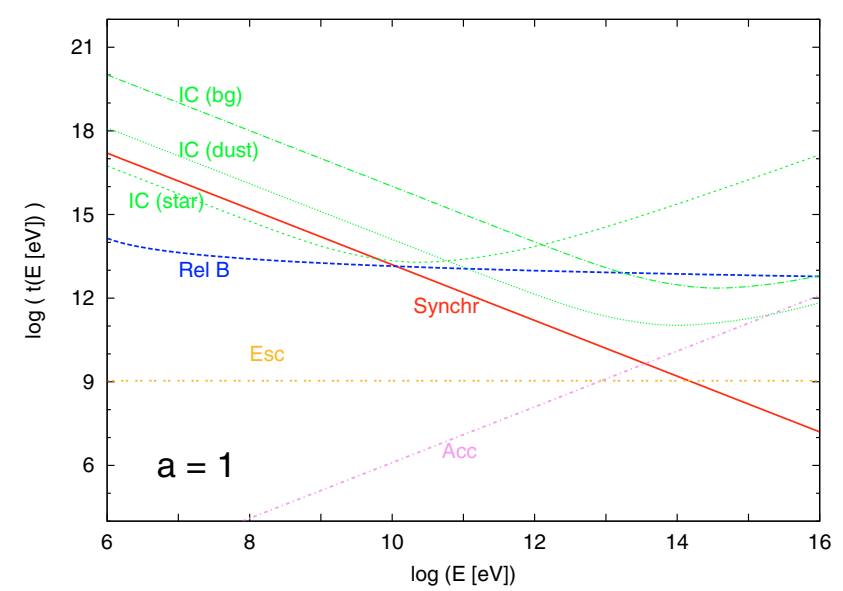

Fig. 4. Acceleration ("Acc"), escape ("Esc"), and cooling times for electrons, due to synchrotron radiation ("Synchr"), due to inverse Compton scattering of dust photons ("IC (dust)"), stellar photons ("IC (star)"), and backgroud photons ("IC (bg)"). Cooling time for relativistic Bremsstrahlung radiation indicated as "Rel B". The figure is for the case with equal energy density in electrons and protons ( $a=1$, see text).

three contributions or addends:

$$
\begin{aligned}
u= & \int E_{e 1} n_{e 1}\left(E_{e 1}\right) \mathrm{d} E_{e 1} \\
& +\int E_{p} n_{p}\left(E_{p}\right) \mathrm{d} E_{p}+\int E_{e 2} n_{e 2}\left(E_{e 2}\right) \mathrm{d} E_{e 2},
\end{aligned}
$$

where $e 1, p$, and $e 2$ stand for relativistic primary electrons, protons, and secondary electron-positron pairs (i.e. pairs coming from charged pion decays), respectively, and $n$ is the number density. The relation between primary electrons and protons is $u_{p}=a u_{e 1}$, with $a \geq 0$. Three cases were considered: $a=0$ (just electrons), $a=1$ (equal energy density in both species), and $a=100$ (proton-dominated case, as observed in the galactic cosmic rays). The order of magnitude of the equipartition magnetic field led to $B \sim 5 \times 10^{-5} \mathrm{G}$ (three times this value for the case $a=100$ ). The maximum value for the energy of the particles was determined through the balance of energy gain and losses. The loss mechanisms considered were (i) synchrotron radiation; (ii) relativistic Bremsstrahlung; (iii) particle escape from the radiation region due to convection by the stellar wind; and (iv) inverse Compton (IC) scattering of IR, stellar and cosmic microwave background photons. In the case of protons, the only relevant losses are proton-proton $(p p)$ inelastic collisions and convective escape. Diffusion is negligible in comparison to convection in this situation. Both primary electrons and protons reach energies up to $\sim 10^{13} \mathrm{eV}$, which is imposed by nonradiative losses, except for $a=100$, where synchrotron losses dominate for electrons. In Fig. 4 we show the losses for electrons in the case $a=1$. Values of magnetic field and maximum energies obtained for electrons and protons are given in Table 1.

\section{Discussion and perspectives}

The presence of highly relativistic particles in a dense medium with high photon density can result in the efficient generation of gamma-rays. Although protons can be effectively accelerated up to the highest energies only in the shocked wind, where the density is low, they can diffuse or be convected upstream up to the region with the swept material and densities of $n \sim 100 \mathrm{~cm}^{-3}$. 
Table 1. Magnetic field and maximum energies calculated for the three cases of $a$ (see text) for primary electrons and maximum energy for protons.

\begin{tabular}{cccc}
\hline \hline$a$ & $\begin{array}{c}B \\
{[\mathrm{G}]}\end{array}$ & $\begin{array}{c}E_{\max }^{e 1} \\
{[\mathrm{eV}]}\end{array}$ & $\begin{array}{c}E_{\max }^{p} \\
{[\mathrm{eV}]}\end{array}$ \\
\hline 0 & $4.0 \times 10^{-5}$ & $8 \times 10^{12}$ & - \\
1 & $5.0 \times 10^{-5}$ & $9 \times 10^{12}$ & $9 \times 10^{12}$ \\
100 & $1.4 \times 10^{-4}$ & $2 \times 10^{13}$ & $3 \times 10^{13}$ \\
\hline
\end{tabular}

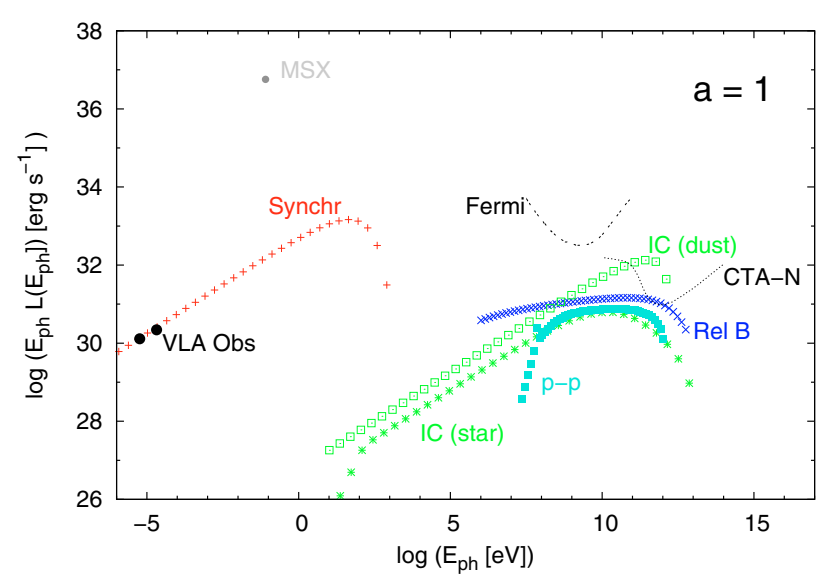

Fig. 5. Spectral energy distribution for the case where the density of primary electrons is equal to the density of protons $(a=1)$. Acronyms as in Fig. 4. Measured radio fluxes from VLA observations: "VLA Obs". The MSX luminosity at $D$ band is also represented. The contribution from secondary pairs is negligible in this case, so is not shown here.

The corresponding gamma-ray emissivity can be calculated using the delta-functional approximation (e.g. Aharonian \& Atoyan 2000; Kelner et al. 2006). For the case $a=1$, the total luminosity from $p p$ interactions is similar to what is obtained from relativistic Bremsstrahlung of electrons, since the cross sections are similar. In Fig. 5 we show the spectral energy distribution obtained for the case $a=1$, with all contributions included (synchrotron self-Compton losses are negligible). It can be seen that in this case the inverse Compton up-scattering of IR photons is the major contribution at high energies, with a peak around $100 \mathrm{GeV}$. The detectability of the source by instruments like the Fermi $\gamma$-ray observatory LAT will depend on the actual particle density and the contribution related to the secondary electrons at large $a$. Detailed calculations for a set of main parameters will be given elsewhere. The $p p$ contribution extends well into the $\mathrm{TeV}$ regime, but it is weaker and will be difficult to detect with ground-based Cherenkov telescope arrays like VERITAS or MAGIC II. In contrast, if the relativistic particle contain is proton-dominated $(a=100)$, gamma-rays from neutral pion decay dominate the high-energy spectrum. The planned CTA North observatory might detect the source, easily yielding imformation on the cutoff at high energies. Observations of the spectral slope in this regime can be used to identify the proton content through the luminosity level and the proton spectral index, since it is preserved in the corresponding photon index. Radio polarization data will provide additional information on the magnetic field. Observations of $\mathrm{BD}+43^{\circ} 3654$ with X-ray observatories like XMM-Newton and Chandra can be very useful for determining the cutoff of the synchrotron spectrum, which is directly related to the maximum energy of the electrons. This, in turn, would yield valuable information on the actual value of the magnetic field and the correctness of the equipartition hypothesis.

Acknowledgements. This work was supported by MinCyT - ANPCyT, project number PICT-2007-00848 / Préstamo BID, and by CONICET, project ID 11220090100078. J.M. and G.E.R. acknowledge support by grant AYA200768034-C03-01 and -02 from the Spanish government and FEDER funds. J.M. and G.E.R. are also supported by Plan Andaluz de Investigación, Desarrollo e Innovación of Junta de Andalucía as research group FQM-322 and excellence fund FQM-5418.

\section{References}

Aharonian, F. A., \& Atoyan, A. M. 2000, A\&A, 362, 937

Araudo, A. T., Romero, G. E., Bosch-Ramon, V., \& Paredes, J. M. 2007, A\&A, 476,1289

Comerón, F., \& Pasquali, A. 2007, A\&A, 467, L23

Condon, K. K., Cotton, W. D., Greisen, E. W., et al. 1998, AJ, 115, 1693

Drury, L. 1983, Rep. Progr. Phys., 46, 973

Gies, D., \& Bolton, C. T. 1986, ApJS, 61, 419

Gvaramadze, V. V., \& Bomans, D. J. 2008, A\&A, 485, L29

Griffith, M., Langston, G., Hefflin, M., et al. 1991, ApJS, 75, 801

Ginzburg, V. L., \& Syrovatskii, S. I. 1964, The Origin of Cosmic Rays (New York: Pergamon Press)

Hanson, M. M. 2003, ApJ, 597, 957

Hoogerwerf, R., de Bruijne, J. H. J., \& de Zeeuw, P. T. 2000, ApJ, 544, L133

Hoogerwerf, R., de Bruijne, J. H. J., \& de Zeeuw, P. T. 2001, A\&A, 365, 49

Kelner, S. R., Aharonian, F. A., \& Bugayov, V. V. 2006, Phys. Rev. D, 74, 4081

Kobulnicky, H. A., Gilbert, I. J., \& Kiminki, D. C. 2010, ApJ, 710, 549

Maíz-Apellániz, J., Walborn, N. R., Galué, H. A., \& Wei, L. H. 2004, ApJS, 151, 103

Noriega-Crespo, A., Van Buren, D., \& Dgani, R. 1997, AJ, 113, 780

Peri, C. S., Benaglia, P., Romero, G. E., \& Martí, J. 2010, Rev. Mex Astron. Astrofis. SC, 38, 61

Taylor, A. R., Gibson, S. J., Peracaula, M., et al. 2003, AJ, 125, 3145

Van Buren, D., \& McCray, R. 1988, ApJ, 329, L93

Van Buren, D., Noriega-Crespo, A., \& Dgani, R. 1995, AJ, 110, 2914 